\title{
Les sciences et techniques appliquées au développement et l'anthropologie
}

Jean Schmitz

\section{OpenEdition}

1 Journals

Édition électronique

URL : http://journals.openedition.org/apad/348

DOI : 10.4000/apad.348

ISSN : 1950-6929

Éditeur

LIT Verlag

Édition imprimée

Date de publication : 15 décembre 1991

\section{Référence électronique}

Jean Schmitz, «Les sciences et techniques appliquées au développement et l'anthropologie », Bulletin de l'APAD [En ligne], 2 | 1991, mis en ligne le 28 juin 2006, consulté le 08 septembre 2020. URL : http:// journals.openedition.org/apad/348; DOI : https://doi.org/10.4000/apad.348

Ce document a été généré automatiquement le 8 septembre 2020

Bulletin de l'APAD 


\title{
Les sciences et techniques appliquées au développement et l'anthropologie
}

\author{
Jean Schmitz
}

1 Si l'anthropologie est une discipline par définition généraliste il n'en est pas de même des sciences et techniques appliquées au développement qui forment des spécialités que l'on peut rapidement identifier. Il s'agit, par exemple, de l'étude des systèmes de production agricole, de l'agro-foresterie, de l'halieutique (aménagement des pêches artisanales), du pastoralisme, du génie civil (irrigation et aménagements hydro-agricoles), de l'urbanisme ("génie urbain")... Or, à la fin des années 70 et au début des années 80 , intervient une demande à l'endroit de l'anthropologie, qui traduit une évolution plus générale marquée par deux phénomènes: une plus grande interdisciplinarité d'une part, la conversion d'une approche verticale et spécialisée en une approche horizontale traduisant les stratégies des acteurs d'autre part. Avant de prendre comme exemple de réponse à cette demande celle de l'anthropologie du développement américaine, il est nécessaire de comprendre la signification de ces types d'évolution du point de vue même des spécialités du développement.

Interdisciplinarité

2 Ces différents "savoirs-pouvoirs" se sont constitués à l'intersection de plusieurs disciplines scientifiques ou universitaires, d'où sont issus des ingénieurs, des praticiens et des techniciens. Les groupes les mieux organisés forment des corps professionnels, tandis qu'à l'état moléculaire, les individus qui les composent se reconnaissent par le statut mystérieux d'expert ou de consultant. Le découpage même des objets de recherche, dans le champ du "développement", est opéré par la succession historique des disciplines qui les définissent, c'est-à-dire leur "profil disciplinaire".

Ainsi le pastoralisme se situe à l'intersection de plusieurs pratiques scientifiques et interventions techniques (Bernus, 1990). Avec le danger de la peste bovine, ce sont les vétérinaires qui occupent ce champ d'intervention durant l'entre-deux-guerres. Durant les années 1950, les hydro-géologues construisent les forages pour lever la contrainte 
d'abreuvement, ce qui aura les effets pervers qu'on connait : modification des rythmes de transhumance, concentration sur les pâturages voisins des forages... Les vétérinaires, quant à eux, s'enferment dans les stations (Landais, 1990). Avec la sécheresse des années 1970, l'objet principal n'est plus l'animal en tant que tel, ni les ressources hydrauliques en vue d'un accroissement du cheptel, mais les pâturages : la porte entr'ouverte par les agrostologues permettra le transfert des méthodes des agronomes (l'étude des "systèmes de production agricole") qui introduisent les recherches sur les "systèmes d'élevage" (Landais, Lhoste \& Milleville, 1987).

4 Ainsi le profil disciplinaire d'une pratique scientifique est-elle un bon indice de la refonte des problématiques internes et des déplacements significatifs des micro-objets de ces savoirs.

5 Autre exemple : la maladie du sommeil. Maladie parasitaire à virus traquée depuis le début du siècle par les médecins pastoriens dans le corps-même du patient indigène, qualifié de "réservoir à virus", elle devint, après la seconde guerre mondiale, le champ d'investigation des entomologistes médicaux qui, eux, s'intéressaient de façon privilégiée à l'insecte vecteur de la trypanosomiase, la mouche tsé-tsé (Dozon 1985).

6 Il ne suffit donc pas d'interpréter les changements de doctrines - certains diraient de paradigmes - comme le résultat de "crises" affectant l'objet même du développement (surpâturage, overfishing.): il faut aussi y discerner en effet l'émergence d'une nouvelle discipline dans un champ du développement, imposant par là même une refonte de la problématique. Les différents savoirs-pouvoirs du développement sont donc dès le départ des enjeux pluri-disciplinaires et il nous faut élucider les raisons pour lesquelles on rapporte à aujourd'hui cette donnée permanente.

De la station au "réel" ou de l'approche verticale à l'approche horizontale.

7 C'est surtout depuis 1975 que l'on observe l'exigence d'interdisciplinarité conduisant à une ouverture vers les sciences sociales. Il ne s'agit plus seulement d'introduire de nouvelles approches dans tel ou tel secteur du développement, car cet appel aux sciences humaines est l'indice d'un changement dans le dispositif institutionnel de la recherche, entendu comme le rapport entre lieu d'observation (ou d'expérimentation) et secteur d'intervention (ou d'application).

8 En effet, durant la période coloniale, les institutions de recherche étaient en relation, dans beaucoup de cas, avec deux sortes de lieux d'application de la recherche. L'un était situé en continuité, sinon en contiguïté, avec le dispositif de recherche, comme dans le cas de l'hôpital, de l'unité de pêche industrielle, de la ferme modèle. Par exemple, l'ingénieur du génie civil construisait un "casier", un périmètre hydro-agricole dans un lieu en général "vidé" de sa population, en ne s'intéressant pas aux activités "hors casier" ou même à la "petite hydraulique" environnante ; ou encore la recherche effectuée dans les centres océanographiques était destinée à la pêche "industrielle", non à la pêche "artisanale". Dans le second lieu ou secteur d'intervention (les paysans "indigènes", les pêcheurs "artisanaux", les éleveurs "traditionnels"...), s'instauraient des rapports hiérarchisés, autoritaires, voire despotiques : marquages des malades, "boy coton"...

9 Avec la décolonisation au sens large - de l'après-guerre aux années 70 - les institutions du premier type ne peuvent plus être le lieu privilégié de l'application du "savoir" du développement, ce qui va provoquer des modifications profondes dans les doctrines et 
les institutions, comme on va le voir en déclinant les différents "secteurs" qui le composent.

Systèmes de production agricole. On observe ce double changement, méthodologique et institutionnel, dans le domaine de l'agronomie tropicale avec l'introduction des "recherches sur les systèmes de production" et leur diffusion dans les Centres Internationaux de Recherche Agronomique (CIRA) comme l'IRRI, l'ICRISAT...

11 En effet, dans les années 1950, prévalait une approche verticale correspondant à la spécialisation de la recherche par plante cultivée (riz, maïs, mils et sorgho...), héritière des diverses divisions impériales du travail agricole (riz d'Indochine, arachide africaine...). L'augmentation des rendements était recherché à travers l'amélioration variétale des plantes résultant des travaux des biologistes et des généticiens (Couty, 1987). Parallèlement, sur le plan institutionnel, cette approche correspondait à un processus de diffusion des travaux des agronomes "de haut en bas", les résultats de la recherche élaborée en station "descendant" vers les paysans par l'intermédiaire des institutions d'encadrement et de vulgarisation (Gilbert, Norman \& Winch, 1980). Le résultat de ce dispositif de la recherche agronomique ce sera la révolution verte succès en Asie, échec en Afrique (Couty, 1987).

Durant les années 70 apparaît une nouvelle méthodologie "horizontale" qui s'attache à analyser le système de production mis en oeuvre par les familles paysannes, à savoir le choix des spéculations culturales, celles-ci formant un ensemble. Il est significatif que cette approche soit apparue à propos de l'analyse des cultures complantées de la Nigéria, alors que la recherche agronomique de la période précédente préconisait la culture "pure" : durant la période coloniale, sans "semis en ligne", pas de semence... Dans la mesure où les modèles ne s'élaborent plus seulement "en station" (agronomique), mais dans le "réel", chez les paysans, le socio-économiste doit jouer un rôle ex ante pour exhiber les problèmes que se pose le paysan, et servir de médiateur entre ce dernier et l'agronome, et non pas ex post comme auparavant. Aux préoccupations d'optimisation des rendements se substituent durant les années 1980 la recherche de variétés adaptées aux sols et résistantes aux sècheresses, l'amélioration des revenus paysans ou de l'emploi rural. Les préoccupations instrumentalistes de l'agronomie peuvent alors passer au second plan par rapport au souci d'inventaire d'une sorte d'ethno-agronomie (Richards,1985).

13 Actuellement on assiste à un retour vers la "recherche fondamentale" - microbiologie, génie génétique - et donc vers le laboratoire, qui s'accompagne peut-être de la création d'une méthodologie qualifiée de "système de production" mais qui s'apparente plus en réalité à des études de "factibilité"...

Pastoralisme. On retrouve une même courbure dans l'histoire du pastoralisme dont on a déjà parlé plus haut: de 1950 à 1975, les vétérinaires s'enferment dans le modèle "de station" de la "ferme agro-pastorale" (Landais \& Lhoste, 1990). Là aussi la méthodologie des "systèmes d'élevage", un peu sur le modèle de l'agronomie, permettra de sortir de la station pour étudier "en situation", par exemple, la conduite d'un troupeau dans le champ même de la pratique pastorale (Landais, Lhoste, Milleville, 1987).

D'où l'intérêt qui se manifeste à l'époque pour les travaux des anthropologues et des géographes, lors d'une série de rencontres: colloque sur le pastoralisme nomade en 1976 à Paris (UNESCO), création de la "Commission pour les Peuples Nomades" au Canada en 1977, colloque sur "l'avenir des peuples pasteurs" de Nairobi en 1980. Cette dernière conférence constate la position difficilement tenable de l'anthropologue qui 
joue souvent la fonction de bouc émissaire : l'échec d'un projet lui est de toute façon imputé, soit parce qu'il en a été absent dès le départ, soit parce que, présent, il refuse l'avenir radieux du progrès.

16 Agro-foresterie. En matière de gestion des forêts on assiste à des changements de doctrines d'autant plus importants qu'ils remettent en cause des "croyances" pluri-séculaires. Par ailleurs, les changements institutionnels ne concernent plus le lieu et les modalités de la recherche mais plutôt son point d'application.

17 Depuis le XVII ${ }^{\text {ème }}$ siècle, en France, les forestiers issus de corps d'État, envisagent la forêt comme une "usine à bois" (Sigaut, 1977) : bois de marine, charpente... Dans les pays tropicaux colonisés, c'est l'exploitation commerciale des forêts qui a prédominé ainsi que la conservation de "réserves" de bois dans le cadre d'un domaine forestier étatisé qui opère par "classement" des forêts et exclusion des usagers locaux qualifiés dorénavant de "riverains" (Catinot, 1984).

Le domaine forestier étant mis en pièces dans les zones sensibles frappées par la sécheresse, on a commencé, à partir du milieu des années 1970, à mettre l'accent sur la "foresterie sociale", l'apogée de ce mouvement étant atteint en 1978, lors du XVIII ${ }^{\text {ème }}$ congrès de foresterie, qui préconise la "foresterie pour le peuple" (Scudder \& Conelly, 1985). En France, une telle révision déchirante est clairement exposée par un article de l'ancien directeur du CTFT, R. Catinot (1984), qui insiste sur la polyvalence de l'utilisation de la forêt par les populations (bois de chauffe, bois d'œuvre, fourrage aérien, pharmacopée, cueillette) rejoignant sans le savoir le souci des forestiers sociaux qui travaillaient en zone de montagne, en France, à la fin du XIX ${ }^{\text {ème }}$ siècle (Kalaora \& Savoye, 1986) .Inspirés par les travaux de Le Play, ces forestiers avaient déjà une conception totalisante de l'aménagement du territoire, où la connaissance pluridisciplinaire avait donc une large part: s'ils ont échoué en France, on peut néanmoins voir dans l'aménagement du Tennessee, en 1933 aux USA, une réalisation lointaine de leurs ambitions, combinant aménagements lourds et conservation par des projets multiformes.

19 Halieutique. Les études concernant la pêche ont subi une évolution contrastée suivant les domaines (maritime, continental) et les secteurs (industriel, artisanal). Il semble néanmoins qu'on puisse déceler deux invariances depuis le début du siècle : celle de la problématique - l'exploitation d'une ressource vivante, renouvelable et non appropriée tend vers des baisses de rendements lorsqu'elle n'est pas régulée (Verdeaux, 1989) - et surtout ce que l'on pourrait appeler l'interdisciplinarité "interne": les biologistes ou les "naturalistes" les plus importants du Muséum d'Histoire Naturelle (Gruvel, Monod, Daged furent en même temps des anthropologues ou des économistes. En réalité cette indistinction de départ va faire assez vite place aux clivages par domaines et par secteurs et la pluridisciplinarité ne perdurera que dans l'étude de la pêche continentale artisanale avec Monod et Daget.

La pêche maritime reste le domaine privilégié des océanographes, qui mettent au point dans les années 1950-60 des modèles biologiques d'exploitation des stocks. Dès cette époque, la modélisation permet de trouver un terrain commun avec les économistes, d'où l'élaboration, presque en même temps, de modèles bio économiques dans le cadre de centres de recherches océanographiques qui s'équipent de navires, effectuant des "campagnes" d'observations en mer.

21 La pêche "artisanale" maritime n'est envisagée alors que comme "pis aller, complément, mais toujours l'envers de ce qui devrait être: la pêche industrielle" 
(Verdeaux, 1989 ; Chauveau, 1989) .Pourtant les déboires de cette dernière sur les côtes des pays tropicaux ainsi que la vitalité économique de la pêche artisanale maritime allaient provoquer, aux alentours de 1975, l'introduction du développement, des sciences sociales dans ce secteur, en particulier à l'ORSTOM et à l'IFREMER (qui d'ailleurs ont organisé un colloque conjoint en 1989 sur ces sujets) .

Santé. La santé, et plus précisément la maladie et ses représentations, constituent un des champs privilégiés de recherche en anthropologie sociale depuis déjà un certain temps .Dès les années 1950-60, apparaît ,en particulier aux USA, une ethnomédecine (Walter, 1981-82) qui pratique cependant volontiers des simplifications dualistes, sinon ethnocentriques. Depuis une quinzaine d'années, on a souligné, le caractère hétérogène des représentations de la maladie et de la thérapeutique - il n'y a pas forcément implication entre diagnostic et thérapie - et, surtout, l'absence d'autonomie du champ en question (Augé, 1986). Par rapport à l'anthropologie médicale, l'anthropologie de la maladie perd en spécificité ce qu'elle gagne en cohérence par la mise en rapport, dans une société donnée, de la maladie avec divers niveaux sociaux: pratiques et savoirs concernant le corps, élaboration des remèdes et en particulier utilisation des plantes...

Dans le domaine institutionnel, alors même que l'hôpital ou le dispensaire devenaient à leur tour objets d'investigation pour les sociologues, les organisations internationales, et en particulier l'OMS et l'UNICEF, préconisaient la "sortie" de la médecine des institutions bio-médicales en revalorisant les guérisseurs, métamorphosés en "tradi-praticiens", ou en formant des travailleurs para-médicaux villageois. Ce fut lors de la conférence internationale d'Alma-Ata, dans le Kazakhstan soviétique en 1978, organisée conjointement par l'OMS et l'UNICEF, que, par une déclaration solennelle, fut affirmée le lien indissociable entre santé et développement dans le cadre des "soins de santé primaires" regroupant l'éducation, l'alimentation et la nutrition, les problèmes d'approvisionnement et d'assainissement de l'eau, les soins maternels et infantiles...

Ainsi, pendant un moment, a-t-on pu penser que s'effectuait une transition d'une approche verticale - étude de telle ou telle maladie tropicale et ethnomédecine - vers une approche plus "horizontale" - soins de santé primaires et anthropologie des "itinéraires thérapeutiques" (le malade s'adresse aussi bien au guérisseur traditionnel qu'à l'institution médicale : dispensaire, hôpital, et c'est cette succession qui a un sens). On ne peut s'empêcher de rapprocher cette notion de celle "d'itinéraire technique" en agronomie : une opération culturale ne peut se comprendre que si on la resitue, pour en comprendre la fonction, non seulement dans le cycle agricole mais dans tout l'éventail des activités du paysan.

Cette demande à l'égard des sciences sociales provoque toutefois moins d'effet qu'elle ne fait sens : elle est en effet souvent l'indice d'un changement radical de doctrine comme dans les cas de l'agro-foresterie ou de l'halieutique. Ces changements s'accompagnent souvent de l'apparition de nouvelles sous-disciplines qui redéfinissent le contour de telle discipline du développement, comme dans le cas de l'agronomie ou du pastoralisme. Or il est rare de faire appel à l'anthropologie car, bien souvent, l'exigence d'interdisciplinarité ou d'un point de vue holiste a disparu, à moins que l'anthropologie elle-même ne se coule dans le moule de la spécialisation comme cela semble être le cas aux USA.

L'exemple américain 
26 1) L'anthropologie appliquée aux USA jusque dans les années 1970. Dans ce pays l'anthropologie appliquée se développe surtout lorsqu'il y a des situations de "contacts" violents, d'abord à l'intérieur même des Etats-Unis, ensuite dans le monde entier (Rank. 1988). C'est d'abord la défense des droits des Indiens sur leurs terres qui va, à la suite d'un très ancien mouvement, aboutir à la création en 1941 de la Société d'anthropologie appliquée. Après 1945, les anthropologues participent à la formation des officiers qui se destinent à des tâches d'administration, dans le Pacifique principalement. Durant les années 1960, l'ancêtre de l'USAID, l'ICA, comporte une division de "développement communautaire" à destination des pays d'Amérique latine.

Durant les années 1970, le danger de voir l'anthropologie se transformer en ingénierie sociale au service de la sécurité des USA (et donc de la stabilité politique des États alliés de ces derniers), disparaît avec les mésaventures du Projet Camelot qui avait des objectifs anti-insurrectionnel en Amérique latine sur financement de l'armée US, ou celles du Projet Thailandais de lutte contre le trafic de la drogue en organisant l'insurrection des populations tribales du Nord.

28 2) Essor de l'anthropologie du développement à partir des années 1970. On peut dire que l'anthropologie du développement provient de la rencontre de deux sensibilités : l'une, populiste, critique l'aveuglement de l'anthropologie classique qui refuse de constater que les (gens) "traditionnels" sont devenus des "pauvres"; l'autre, écologique, remet en cause la positivité des résultats du "développement".

Dès 1970, sous l'impulsion de Mac Namara en particulier, la Banque Mondiale tente de s'adresser aux rural poors alors qu'elle finançait jusque-là des projets à forte intensité capitalistique. Ces préoccupations nouvelles se concrétisent lors des nouvelles directives du Congrès à l'USAID en 1973. Cela se traduit en termes bureaucratiques à la Banque Mondiale puisque tout projet doit, pour être accepté, faire l'objet d'un diagnostic social (Social soundness analysis ). Néanmoins, en 1981, avec l'arrivée de Reagan à la présidence, la rentabilité revient à l'ordre du jour (Rank, 1988).

C'est autour des années 1985 qu'apparaît le souci de l'environnement dégradé par un "développement destructif" pour reprendre le mot de M. Horowitz, l'un des trois anthropologues (avec T. Scudder et D. Brokensha) de l'Institute for Development Anthropology (I.D.A.) créé en 1976 et lié à l'université de Binghampton (Etat de New York) (Horowitz, 1988).

31 Or, si l'on ramène à trois les conditions de la professionalisation de cette branche de l'anthropologie (Rank, 1988), on s'aperçoit que la première concerne justement la spécialisation sectorielle ou thématique du chercheur selon les disciplines $d u$ développement que nous avons énumérées plus haut. (il faut remarquer au passage que cette spécialisation subit les règles de grammaire du pays d'origine et qu'il n'y a pas stricte correspondance entre les diverses taxinomies nationales de ces spécialités). D'après le personnel de l'IDA, il semble que ce soit-là la condition de la véritable ingénierie sociale, impliquant l'intervention à toutes les étapes du projet. Les deux autres exigences sont déjà du ressort de l'anthropologue : le passage du micro au macro et le comparatisme, d'une part, l'analyse institutionnelle des objectifs latents du projet, d'autre part, n'introduisent pas de rupture avec l'anthropologie tout court.

32 Mais à trop se spécialiser l'anthropologie risque de perdre sa spécificité et de se retrouver dans une situation bloquée. D'un côté le point de vue holiste ou "intégriste", de l'autre le mouvement vers la spécialisation, qui caractérise toute activité 
scientifique, et donc vers le "réductionnisme"... Il y à là néanmoins un objet de réflexion à développer, ne serait-ce qu'au niveau institutionnel, quant à l'organisation interne de l'APAD.

\section{BIBLIOGRAPHIE}

Augé M., 1986, "L'anthropologie de la maladie", l'Homme, XXVI (97-98) :81-93.

Bernus E., 1990, "En guise de conclusion : les pasteurs nomades africains : du mythe éternel aux réalités présentes", Cati. Sci. Hum. , 26 (1-2) :267-280.

Catinot R., 1984, "En Afrique francophone l'avenir forestier tropical se jouera dans le cadre du monde rural (,...)", Bois et forêts tropicaux, 203 ( 1 ) : 7-43.

Couty Ph., 1987, "La production agricole en Afrique sub-saharienne : manières de voir et façons d'agir , Cah.SC1.Hum., 23 (3-4) :3n-408.

Chauveau J -P., 1985, "L'avenir d'une illusion : histoire de la production et des politiques vivrières en Côte d'Ivoire", Etudes Rurales, 99-100 : 281-235

Chauveau J.-P., 1989, "Histoire de la pêche industrielle au Sénégal et politiques d'industrialisation", Cab. Sei. Hum., 25 (1-2) : 237-287.

Diemer G., 1986, "Le génie rural, applicabilité universelle ou ethnoscience ?" in "Aménagements hydro-agricoles et systèmes de production", Actes du III ${ }^{\mathrm{e}}$ séminaire de Montpellier (CIRAD), T1, 175-185

Dozon J.-P., 1985, "Quand les Pastoriens traquaient la maladie du sommeil", Sciences sociales et Santé, III (3-4) : 27-56.

Galaty J.G., Aronson D. \& Salzman P.C., 1981, "L'avenir des peuples pasteurs", Compte-rendu de la Conférence tenue à Nairobi, Kenya 4-6 Août, Ottawa (Ontario), CRDI, 432 p.

Gilbert E.H., Norman D.W \& Finch F.E., 1980, Les recherches sur les systèmes d'exploitation agricole : une évaluation critique, Michigan, Michigan State University, 150 p. multigr. ("Cahiers MSU" 6).

Horowitz M., 1987, "Destructive development ", Development Anthropology Network, Bulletin of the Institute for Development Anthropology, 5 (I) :1-3.

1988, "Anthropology and the New Development Agenda", Development Anthropology Network, Bulletin of the I.D A., 6 (1) : 1-4.

Kalaora B., \& Savoye A., 1986 La forêt pacifiée. Les forestiers de l'École de Le Play défenseurs des populations de montagne (1860-1913), Paris, L'Harmattan.

Landais E., 1990, "Sur les doctrines des vétérinaires coloniaux français en Afrique noire", Cab. Sci. Hum., 26 (1-2) : 33-71.

Landais E, Lhoste Ph. \& Milleville P., 1987, "Points de vue sur la zootechnie et les systèmes d'élevage tropicaux", cab.Sci.Hum., 23 (3-4) : 421-439. 
Marie M., 1984, "Pour une anthropologie des grands ouvrages : le canal de Provence", Annales de la recherche urbaine ,21:5- 35 .

Pouillon F., 1990,. "Sociétés pastorales et développement : histoire des politiques et critique des doctrines , Cab. Sci. Hum. 26(1-2) : 3-7.

Rank E., 1988, "L'anthropologie du développement aux États Unis : force et promesses d'une nouvelle profession", Cab. -sci. Hum. 24 (4) : 453-469.

Richards P., 1985, "Indigenous Agricultural Revolution", Londres, Hutchinson.

Scudder T., \& Connely T., 1985, "Systèmes d'aménagement de la pêche fluviale", Rome, FAO, 63 p. multigr. ("Doc. techniques pêche, 263).

Sigaut F., 1977, "Les savoirs relatifs aux animaux domestiques, leur rôle historique et technologique", Ethnozootechnie, 20, 16-22.

Thoenig J.C., \& Friedberg E., 1969, "Politiques urbaines et stratégies corporatives", Politique urbaine, 4, (oct.- dec.) : 387-412.

Walter A., 1981-1982, "Ethnomédecine et anthropologie médicale : bilan et perspectives", Cab.ORSTOM , ser, Sci. Hum., XVIII (4) : 405-414.

\section{AUTEUR}

JEAN SCHMITZ

ORSTOM, Paris 